\title{
Clinicopathological differences in signet ring cell adenocarcinoma between early and advanced gastric cancer
}

\author{
Yi-Chu Kao ${ }^{1,2} \cdot$ Wen-Liang Fang ${ }^{1,2} \cdot$ Ruei-Fang Wang ${ }^{3} \cdot$ Anna Fen-Yau $\mathrm{Li}^{2,4} \cdot$ Muh-Hwa Yang ${ }^{5,6} \cdot$ Chew-Wun Wu ${ }^{1,2}$. \\ Yi-Ming Shyr ${ }^{1,2} \cdot$ Kuo-Hung Huang ${ }^{1,5}$ (i)
}

Received: 27 February 2018 / Accepted: 27 July 2018 / Published online: 1 August 2018

(c) The International Gastric Cancer Association and The Japanese Gastric Cancer Association 2018

\begin{abstract}
Background Signet ring cell adenocarcinoma is a histological classification based on the WHO classification. The presence of this specific histological type is associated with a worse pathological appearance. The prognosis of signet ring cell adenocarcinoma in gastric cancer patients after curative surgery is still under debate.

Methods From January 1988 to December 2012, a total of 2971 patients, including 819 early and 2152 advanced gastric cancer patients underwent curative resection for gastric cancer. Among them, there were 185 cases of signet ring cell adenocarcinoma in early gastric cancer patients, while there were 570 cases in advanced gastric cancer patients.

Results The overall incidence of signet ring cell adenocarcinoma was $25.4 \%$. Our results showed that the 5-year overall survival rates of early gastric cancer patients with signet ring cell adenocarcinoma and non-signet ring cell adenocarcinoma were 90.7 and $83.2 \%$, respectively $(P=0.001)$. The 5 -year disease-free survival rates of early gastric cancer patients with signet ring cell adenocarcinoma and non-signet ring cell adenocarcinoma were 87.4 and $81.6 \%$, respectively $(P=0.003)$. The 5-year overall survival rates of advanced gastric cancer patients with signet ring cell adenocarcinoma and non-signet ring cell adenocarcinoma were 32.1 and $37.9 \%$, respectively $(P=0.041)$. The 5-year disease-free survival rates of advanced gastric cancer patients with signet ring cell adenocarcinoma and non-signet ring cell adenocarcinoma were 28.6 and $35.2 \%$, respectively $(P=0.037)$. Signet ring cell adenocarcinoma was an independent predictor for overall survival in advanced gastric cancer $(P=0.017)$.

Conclusion The clinical features and prognosis of signet ring cell adenocarcinoma are different between early and advanced gastric cancer. Signet ring cell adenocarcinoma is a poor prognostic factor in advanced gastric cancer after curative resection.
\end{abstract}

Keywords Gastric cancer · Gastrectomy $\cdot$ Signet ring cell adenocarcinoma

Kuo-Hung Huang

khhuang@vghtpe.gov.tw

1 Division of General Surgery, Department of Surgery, Taipei Veterans General Hospital, No. 201, Sec. 2, Shipai Rd., Beitou District, Taipei City 11217, Taiwan

2 School of Medicine, National Yang-Ming University, Taipei, Taiwan

3 Department of Emergency Medicine, Taipei City Hospital, Ren-Ai Branch, Taipei, Taiwan

4 Department of Pathology, Taipei Veterans General Hospital, Taipei, Taiwan

5 School of Medicine, Institute of Clinical Medicine, National Yang-Ming University, Taipei, Taiwan

6 Division of Medical Oncology, Department of Oncology, Taipei Veterans General Hospital, Taipei, Taiwan

\section{Introduction}

Gastric cancer is the fifth most common cancer and the third most common cause of cancer-related death worldwide [1]. Although the prevalence of gastric cancer has gradually declined, the incidence of signet ring cell carcinoma has gradually increased. Signet ring cell adenocarcinoma is defined as poorly cohesive malignant tumor cells with prominent mucin in the cytoplasm and eccentric crescentshaped nuclei. There are several pathological classifications designed for evaluating tumor biological behavior in gastric cancer. According to the Japanese Gastric Cancer Association [2], signet ring cell carcinoma is defined as an undifferentiated carcinoma. Lauren's classification is currently the most useful and widely applied scheme for gastric cancer [3]. Generally, signet ring cell carcinoma is classified as a 
diffuse type of gastric cancer by definition. In the WHO classification [4] of gastric cancer histological classification, gastric cancer was classified as papillary, tubular, mucinous, signet ring cell, and undifferentiated adenocarcinoma. Signet ring cell adenocarcinoma was defined as having more than $50 \%$ of gastric cancer cells. Signet ring cell adenocarcinoma is usually thought to have a poorer prognosis $[5,6]$.

Early gastric cancer is defined as a gastric tumor that has invaded no more deeply than the submucosa layer (T1), irrespective of the lymph node metastasis status. Advanced gastric cancer is defined as a tumor that has invaded as least to the muscle layer (T2-T4). The prognosis of signet ring cell carcinoma in early gastric cancer and advanced gastric cancer is still controversial in the literature, especially the prognosis in early gastric cancer. This study aims to investigate the clinicopathological evidence and variations in signet ring cell adenocarcinoma in early and advanced gastric cancer patients.

\section{Patients and methods}

From January 1988 to December 2012, a total of 2971 patients diagnosed with gastric cancer underwent curative resection for gastric cancer in the Department of Surgery at Taipei Veterans General Hospital. Prior to surgery, chest radiography, upper gastrointestinal (UGI) endoscopy, and computed tomography (CT) were performed. Patients with definitive evidence of distant organ metastasis or peritoneal seeding did not undergo surgical intervention and were referred to oncologists for evaluation for chemotherapy instead. After laparotomy, peritoneal lavage cytology was collected from each patient. Subtotal gastrectomy was performed for distal or middle third lesions, while total gastrectomy was performed for proximal third lesions. Standard D2 lymph node dissection [7] was performed in patients with curative intent. Patients who could not undergo gastric resection, including bypass surgery or exploratory laparotomy alone, were excluded from this study. All the surgical specimens were examined by experienced pathologists. The gross features of the pathological specimens were evaluated according to the tumor location, tumor size, and Borrmann's classification. Borrmann's classification includes a superficial type, Borrmann type I (polypoid tumor), Borrmann type II (ulcerated tumor with sharp demarcated margin), Borrmann type III (ulcerated tumor without demarcated margins that infiltrates to the surrounding gastric wall), and Borrmann type IV (diffuse infiltrating tumor). Borrmann type I and II tumors are well-defined tumor (localized type), while Borrmann type III and IV tumors have an ill-defined appearance (infiltrating type). The microscopic features of histology, pathology and cell differentiation were analyzed according to the cell grade of tumor differentiation, the stromal reaction type (medullary, intermediate and scirrhous types), Ming's histological classification [8] (expanding or infiltrating type), and lymphovascular invasion patterns. Patients with positive cytology findings after surgery classified as having M1 disease. The staging systems were based on the 8th edition of the American Joint Committee (AJCC) TNM classification for gastric cancer [9].

After surgery, patients were regularly followed up at the outpatient department every 3 months. Recurrence was defined as the first sign of tumor relapse on imaging, cytological analysis of ascites obtained by abdominocentesis, endoscopic findings of tumor recurrence, and/or metastases identified on a bone scan. The relapse pattern of tumor recurrence was recorded in detail. The follow-up data were prospectively collected and regularly updated. The overall survival (OS) was defined from the date of operation to the date of death or the latest follow-up. The disease-free survival (DFS) was defined as the length of period after surgery for gastric cancer during which the patient survived without recurrence. The recurrent patterns in our series were defined as locoregional recurrence, peritoneal dissemination, distant lymph node metastasis and distant organ (hematogenous) metastasis. Patients could have more than one metastasis at the time of initial diagnosis of recurrence.

In early gastric cancer, signet ring cell adenocarcinoma was defined as more than $50 \%$ cancer cells in the mucosa. In advanced gastric cancer, signet ring cell was defined as more than $50 \%$ cancer cells in the mucosa irrespective of the deep invasive component. When signet ring cell adenocarcinoma invaded beyond the submucosal layer, the deep invasive component of advanced signet cell carcinoma commonly includes poorly cohesive carcinoma cells with scant mucin [10]. If the mucosa is lost by ulceration, this kind of gastric cancer was classified as non-signet cell adenocarcinoma. Figure 1a shows a pure signet ring cell adenocarcinoma in early gastric cancer while Fig. $1 \mathrm{~b}$ reveals an advanced signet ring cell containing adenocarcinoma in the deep invasive part. This study was approved by the Institutional Review Board from Taipei Veterans General Hospital (IRB-TPEVGH No. 2017-05-004BC).

All statistical analyses were carried out using SPSS software version 18.0 (SPSS Inc., Chicago, IL USA). Clinicopathological differences were compared with the Chi-square test. Survival was evaluated with a Kaplan-Meier curve analysis and log rank test. Prognostic factors were evaluated with a Cox regression model. A $P$ value $<0.05$ was considered statistically significant.

\section{Results}

Among all these gastric cancer patients, $25.4 \%$ had signet ring cell adenocarcinoma, including $22.6 \%$ of patients in early gastric cancer and $26.5 \%$ of patients in advanced 
$\mathbf{a}$

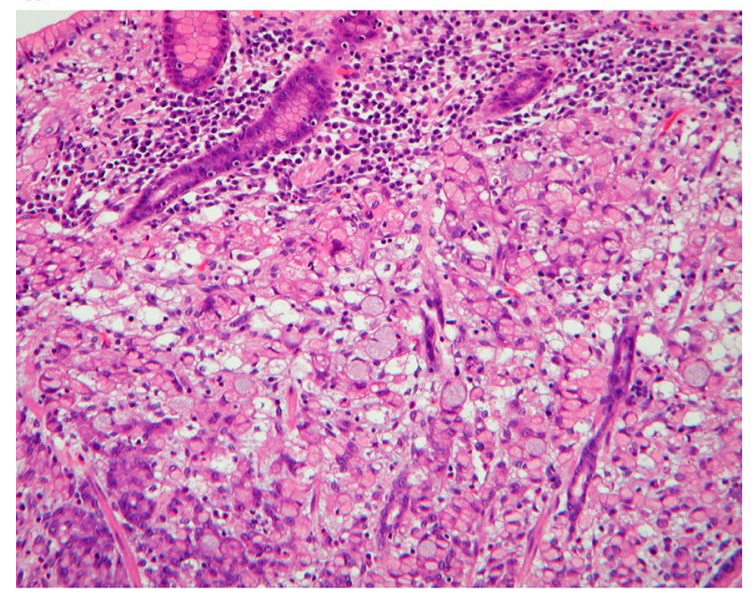

b

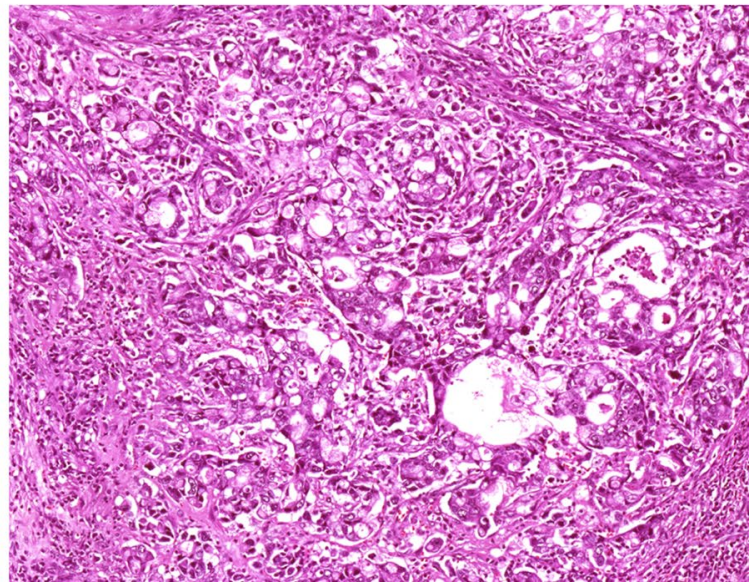

Fig. 1 Microscopic feature of signet ring cell adenocarcinoma and the picture of deeply invasive part of advanced signet ring cell adenocarcinoma. a Pure signet ring cell adenocarcinoma in early gastric can-

gastric cancer. In patients with early gastric cancer, the clinicopathological characteristics suggest that gastric cancer patients with signet ring cell adenocarcinoma were younger $(P<0.001)$, had a greater female/male ratio $(P<0.001)$, had a smaller tumor size $(P<0.001)$, had predominant involvement of the middle stomach $(P<0.001)$, and had more poorly differentiated disease $(P<0.001$, Table 1$)$.

In patients with advanced gastric cancer, the clinicopathological characteristics indicate that gastric cancer patients with signet ring cell adenocarcinoma were younger $(P<0.001)$, had a greater female/male ratio $(P<0.001)$, had a larger tumor size $(P=0.001)$, had predominant involvement of the middle stomach $(P<0.001)$, had more poorly differentiated disease $(P<0.001)$, had a more advanced Borrmann type $(P<0.001)$, had more scirrhous-type stromal reaction $(P<0.001)$, were more commonly infiltrating type based on Ming's histology $(P<0.001)$, had more lymphovascular invasion $(P<0.001)$, had a greater tumor invasion depth $(P<0.001)$ and had more lymph node metastasis $(P<0.001$, Table 2$)$.

The 5-year overall survival rates of early gastric cancer patients with signet ring cell adenocarcinoma and non-signet ring cell adenocarcinoma were 90.7 and $83.2 \%$, respectively $(P=0.001)$. The 5 -year disease-free survival rates of early gastric cancer patients with signet ring cell adenocarcinoma and non-signet ring cell adenocarcinoma were 87.4 and $81.6 \%$, respectively $(P=0.003)$. The 5 -year overall survival rates of advanced gastric cancer patients with signet ring cell adenocarcinoma and non-signet ring cell adenocarcinoma are 32.1 and $37.9 \%$, respectively $(P=0.041)$. The 5 -year disease-free survival rates of advanced gastric cancer patients with signet ring cell adenocarcinoma and non-signet ring cell adenocarcinoma were 28.6 and $35.2 \%$, respectively cer. $\mathbf{b}$ When the deep invasive part is associated with signet ring cell containing adenocarcinoma, the tumor is regarded as advanced signet ring cell adenocarcinoma

$(P=0.037)$. Interestingly, the survival rates of gastric cancer patients with signet ring cell adenocarcinoma between those with early gastric cancer and those with advanced gastric cancer were different than those of patients with non-signet ring cell adenocarcinoma (Fig. 2).

In early gastric cancer, multivariate analysis with overall survival as an endpoint revealed that age $(P<0.001)$ and pathological $\mathrm{N}$ category $(P=0.005)$ were significantly correlated in gastric cancer. Lymph node status was an independent prognostic factor for early gastric cancer with signet ring cell adenocarcinoma. However, signet ring cell adenocarcinoma was not an independent predictor for overall survival in early gastric cancer $(P=0.510$, hazard ratio: $0.827,95 \%$ confidence interval: 0.471-1.454, Table 3).

In advanced gastric cancer, a multivariate analysis with overall survival as an endpoint showed that age $(P<0.001)$, tumor size $(P<0.001)$, Borrmann classification $(P<0.001)$, lymphovascular invasion $(P<0.001)$, pathological T category $(P<0.001)$, pathological $\mathrm{N}$ category $(P<0.001)$ and signet ring cell adenocarcinoma $(P=0.017)$ were significantly correlated with gastric cancer. Signet ring cell adenocarcinoma was an independent predictor for overall survival in advanced gastric cancer $(P=0.017$, hazard ratio: 0.878 , 95\% confidence interval: 0.782-0.973, Table 3 ).

Furthermore, we evaluated the prognosis of signet ring cell gastric cancer in the subgroups of different stages. The results showed that gastric cancer patients with signet ring cell adenocarcinoma had significantly better survival than those with non-signet ring cell adenocarcinoma in stage I disease $(P<0.001)$. However, the survival difference between signet ring cell and non-signet ring cell adenocarcinoma was not obvious in stage II $(P=0.487)$ and stage III gastric cancer $(P=0.231$, Fig. 3$)$. 
Table 1 Clinicopathological characteristics of 819 early gastric cancer patients with and without signet ring cell carcinoma

\begin{tabular}{llll}
\hline Variable & $\begin{array}{l}\text { Signet } \\
\text { ring cell } \\
(n=185)\end{array}$ & $\begin{array}{l}\text { Non-signet } \\
\text { ring cell } \\
(n=634)\end{array}$ & $P$ value \\
\hline
\end{tabular}

Age (years)
$<65$
$\geqq 65$
Sex
Male
Female
Tumor size $(\mathrm{cm})$
$<4$
$4-8$
$>8$

Tumor location

Upper stomach

Middle stomach

125

60

96

89

119

59

7

Lower stomach

Whole stomach

Gland-forming differentia-

tion

Poorly

Moderately

Well

Stromal reaction type

Medullary type

Intermediate type

Scirrhous type

Ming's

Expanding

Infiltrating

Lymphovascular invasion

No

Yes

TNM pathological $\mathrm{N}$

category $^{\mathrm{a}}$

N0
N1
N3

\section{AJCC TNM stage}

II

III

Overall survival rate (5 year)

Disease-free survival rate (5 year)

$* P<0.05$, statistical significance

${ }^{a}$ American Joint Committee on Cancer (AJCC), Cancer Staging Manual, eighth edition, T category: T1, T2, T3, T4; N category: N0, N1, $\mathrm{N} 2, \mathrm{~N} 3$
In terms of recurrence after surgery, the overall recurrence rates of gastric cancer patients with and without signet ring cell adenocarcinoma were 26.3 and $18.6 \%$, respectively $(P<0.001)$. Considering the recurrent patterns, the rates of locoregional recurrence in signet and non-signet ring cell adenocarcinoma were $69 / 755(9.1 \%)$ vs. $167 / 2216(7.5 \%)$, respectively $(P=0.160)$. The recurrence rates of peritoneal seeding in signet and non-signet ring cell adenocarcinoma were $130 / 755(17.2 \%)$ vs. $153 / 2216(6.9 \%)$, respectively $(P<0.001)$. The recurrence rates of distant lymph node metastasis in signet and non-signet ring cell adenocarcinoma were $57 / 755(7.5 \%)$ vs. $83 / 2216(3.7 \%)$, respectively $(P<0.001)$. The recurrence rates of distant organ metastasis in signet and non-signet ring cell adenocarcinoma were $61 / 755(8.1 \%)$ vs. $209 / 2216(9.4 \%)$, respectively ( $P=0.264)$. The rates of peritoneal dissemination and distant lymph node metastasis in patients with signet ring adenocarcinoma were significantly higher than in patients with nonsignet ring cell adenocarcinoma. There was no significant difference in recurrence rate between signet ring cell and non-signet ring cell (5.94\% vs. $5.21 \%, P=0.694)$ in early gastric cancer. However, there was significant higher recurrence rate in patients with signet ring cell adenocarcinoma than in patients with non-signet ring cell adenocarcinoma in advanced gastric cancer $(32.9 \%$ vs. $24.0 \%, P<0.001$, Table 4). Signet ring cell adenocarcinoma displayed an increased significance in recurrence and mortality (DFS: $P=0.009$, hazard ratio: $0.859,95 \%$ confidence interval: $0.769-0.968$ ) in advanced gastric cancer but not in early gastric cancer instead (DFS: $P=0.399$, hazard ratio: 1.154 , 95\% confidence interval: 0.827-1.610).

\section{Discussion}

Our studies found that patients with signet ring cell adenocarcinoma were younger in age than non-signet ring cell adenocarcinoma. Besides, we observed a higher female/male ratio among patients with signet ring cell adenocarcinoma than among those with non-signet ring cell adenocarcinoma. This phenomenon was also reported in other series [11, 12]. Kwon [13] reported that signet ring cell gastric cancer was located in middle stomach in early gastric cancer and in the lower stomach in advanced gastric cancer. Compared with their studies, we found that signet ring cell adenocarcinoma tended to be located in middle stomach both in early and advanced gastric cancer. Although signet ring cell adenocarcinoma has relatively poor clinicopathological features compared with non-signet cell adenocarcinoma, the prognosis of signet ring cell adenocarcinoma is better in early gastric cancer. Nevertheless, patients with signet ring cell adenocarcinoma have a poor prognosis in advanced gastric cancer. Furthermore, we found that signet ring cell adenocarcinoma 
Table 2 Clinicopathological characteristics of 2152 advanced gastric cancer patients with and without signet ring cell carcinoma

\begin{tabular}{|c|c|c|c|}
\hline Variable & $\begin{array}{l}\text { Signet ring cell } \\
(n=570)\end{array}$ & $\begin{array}{l}\text { Non-signet ring cell } \\
(n=1582)\end{array}$ & $P$ value \\
\hline \multicolumn{4}{|l|}{ Age (years) } \\
\hline$<65$ & 336 & 465 & \\
\hline$\geqq 65$ & 234 & 1117 & $<0.001^{*}$ \\
\hline \multicolumn{4}{|l|}{ Sex } \\
\hline Male & 346 & 1311 & \\
\hline Female & 224 & 271 & $<0.001 *$ \\
\hline \multicolumn{4}{|l|}{ Tumor size $(\mathrm{cm})$} \\
\hline$<4$ & 96 & 308 & \\
\hline $4-8$ & 288 & 883 & \\
\hline$>8$ & 186 & 391 & $0.001^{*}$ \\
\hline \multicolumn{4}{|l|}{ Tumor location } \\
\hline Upper stomach & 87 & 315 & \\
\hline Middle stomach & 223 & 451 & \\
\hline Lower stomach & 205 & 758 & \\
\hline Whole stomach & 55 & 58 & $<0.001^{*}$ \\
\hline \multicolumn{4}{|l|}{ Gross appearance } \\
\hline Borrmann type I and II & 82 & 498 & \\
\hline Borrmann type III and IV & 488 & 1084 & $<0.001^{*}$ \\
\hline \multicolumn{4}{|l|}{ Gland-forming differentiation } \\
\hline Poorly & 570 & 749 & \\
\hline Moderately & 0 & 802 & \\
\hline Well & 0 & 31 & $<0.001 *$ \\
\hline \multicolumn{4}{|l|}{ Stromal reaction type } \\
\hline Medullary type & 18 & 191 & \\
\hline Intermediate type & 139 & 1071 & \\
\hline Scirrhous type & 413 & 320 & $<0.001^{*}$ \\
\hline \multicolumn{4}{|l|}{ Ming's } \\
\hline Expanding & 35 & 367 & \\
\hline Infiltrating & 535 & 1215 & $<0.001^{*}$ \\
\hline \multicolumn{4}{|l|}{ Lymphovascular invasion } \\
\hline No & 135 & 320 & \\
\hline Yes & 435 & 1262 & 0.083 \\
\hline \multicolumn{4}{|l|}{ TNM pathological T category } \\
\hline $\mathrm{T} 2$ & 74 & 402 & \\
\hline $\mathrm{T} 3$ & 411 & 911 & \\
\hline $\mathrm{T} 4$ & 85 & 269 & $<0.001 *$ \\
\hline \multicolumn{4}{|l|}{ TNM pathological $\mathrm{N}$ category } \\
\hline No & 111 & 408 & \\
\hline N1 & 84 & 209 & \\
\hline $\mathrm{N} 2$ & 96 & 369 & \\
\hline $\mathrm{N} 3$ & 279 & 596 & $<0.001 *$ \\
\hline \multicolumn{4}{|l|}{ AJCC TNM stage } \\
\hline I & 28 & 210 & \\
\hline II & 158 & 405 & \\
\hline III & 384 & 967 & $<0.001^{*}$ \\
\hline Overall survival rate ( 5 year) & $32.1 \%$ & $37.9 \%$ & $0.041^{*}$ \\
\hline Disease-free survival rate (5 year) & $28.6 \%$ & $35.2 \%$ & $0.037 *$ \\
\hline
\end{tabular}

$* P<0.05$, statistical significance 

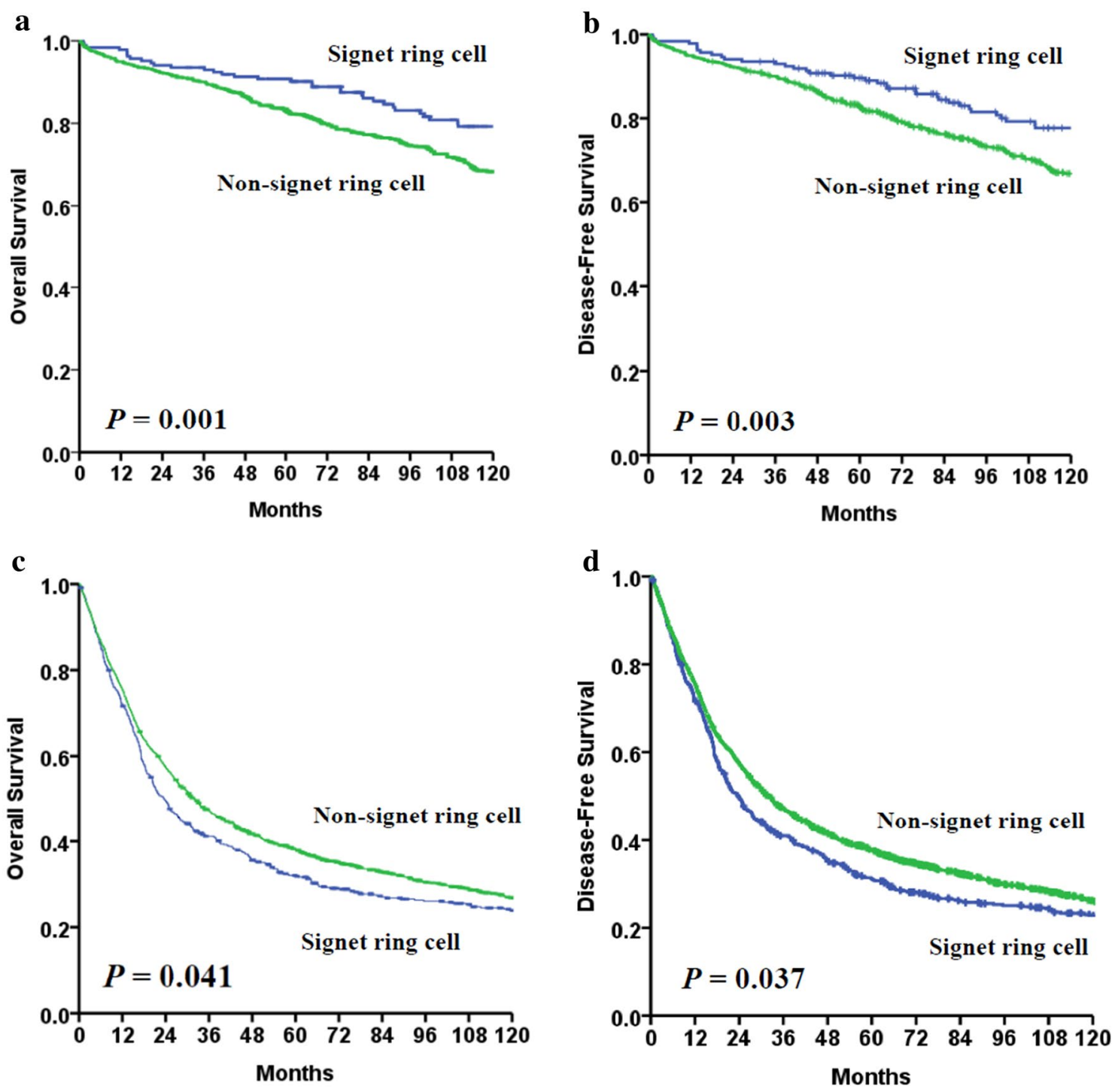

Fig. 2 a Overall survival of early gastric cancer patients with signet and non-signet ring cell adenocarcinoma in months, $P=0.001$ (log rank test). b Disease-free survival of early gastric cancer patients with signet and non-signet ring cell adenocarcinoma in months, $P=0.003$ (log rank test). c Overall survival of advanced gastric cancer patients

was an independent predictor of a poorer prognosis factor in advanced gastric cancer.

In terms of the prognosis of signet ring cell adenocarcinoma, Maehara et al. [14] and Kim et al. [15] reported that signet ring cell carcinoma has similar prognosis in both early and advanced gastric cancer. Jiang et al. [16] reported that signet ring cell carcinoma is associated with better survival than non-signet ring carcinoma in early gastric cancer but these two types of carcinoma have a similar prognosis in advanced gastric cancer. For signet ring cell carcinoma in early gastric cancer, Ha et al. [17] and Hyung et al. [18] reported that early signet ring cell carcinoma has better prognosis than non-signet ring cell

with signet and non-signet ring cell adenocarcinoma in months, $P=0.041$ (log rank test). d Disease-free survival months of advanced gastric cancer patients with signet and non-signet ring cell adenocarcinoma in months, $P=0.037$ (log rank test)

carcinoma. However, there are still some studies that have shown that the prognosis is similar between early signet ring cell carcinoma and non-signet ring cell carcinoma $[14,15,19]$. For advanced gastric cancer, several studies showed a similar prognosis between signet cell carcinoma and non-signet ring cell carcinoma $[11,15$, 16]. Taghavi et al. [20] reported a study with more than $>10,000$ gastric cancer patients in United States. This registry cohort study showed no significant prognostic difference between signet ring cell carcinoma and nonsignet cell carcinoma in advanced gastric cancer. Another study by Li et al. [21] evaluated a population in Korea, which reported worse prognosis in advanced signet ring 
Table 3 Multivariate analysis with overall survival as an endpoint in early and advanced gastric cancer patients

\begin{tabular}{|c|c|c|c|c|c|c|}
\hline \multirow[t]{2}{*}{ Variables } & \multicolumn{3}{|c|}{ Early gastric cancer } & \multicolumn{3}{|c|}{ Advanced gastric cancer } \\
\hline & $P$ value & Hazard ratio & $95 \%$ confidence interval & $P$ value & Hazard ratio & $95 \%$ confidence interval \\
\hline Age & $<0.001 *$ & 4.033 & $2.961-5.493$ & $<0.001 *$ & 1.451 & $1.302-1.667$ \\
\hline Tumor size & 0.856 & 0.979 & $0.775-1.235$ & $<0.001^{*}$ & 1.201 & $1.052-1.243$ \\
\hline Tumor location & 0.679 & 0.966 & $0.820-1.138$ & 0.272 & 1.025 & $0.934-1.123$ \\
\hline Borrmann classification & - & - & - & $<0.001^{*}$ & 1.421 & $1.165-1.459$ \\
\hline Cell grade & 0.488 & 0.896 & $0.658-1.221$ & 0.821 & 1.023 & $0.872-1.183$ \\
\hline Stromal reaction & 0.696 & 1.042 & $0.849-1.278$ & 0.572 & 1.059 & $0.942-1.137$ \\
\hline Ming's classification & 0.645 & 1.071 & $0.799-1.435$ & 0.563 & 0.922 & $0.827-1.125$ \\
\hline Lymphovascular invasion & 0.871 & 1.027 & $0.747-1.411$ & 0.073 & 1.203 & $0.998-1.523$ \\
\hline TNM pathologic $\mathrm{T}$ category & - & - & - & $<0.001^{*}$ & 1.301 & $1.222-1.415$ \\
\hline TNM pathologic $\mathrm{N}$ category & $0.005^{*}$ & 1.383 & $1.104-1.732$ & $<0.001 *$ & 1.478 & $1.371-1.554$ \\
\hline Signet ring cell present & 0.510 & 0.827 & $0.471-1.454$ & $0.017 *$ & 0.878 & $0.782-0.973$ \\
\hline
\end{tabular}

$* P<0.05$, statistical significance
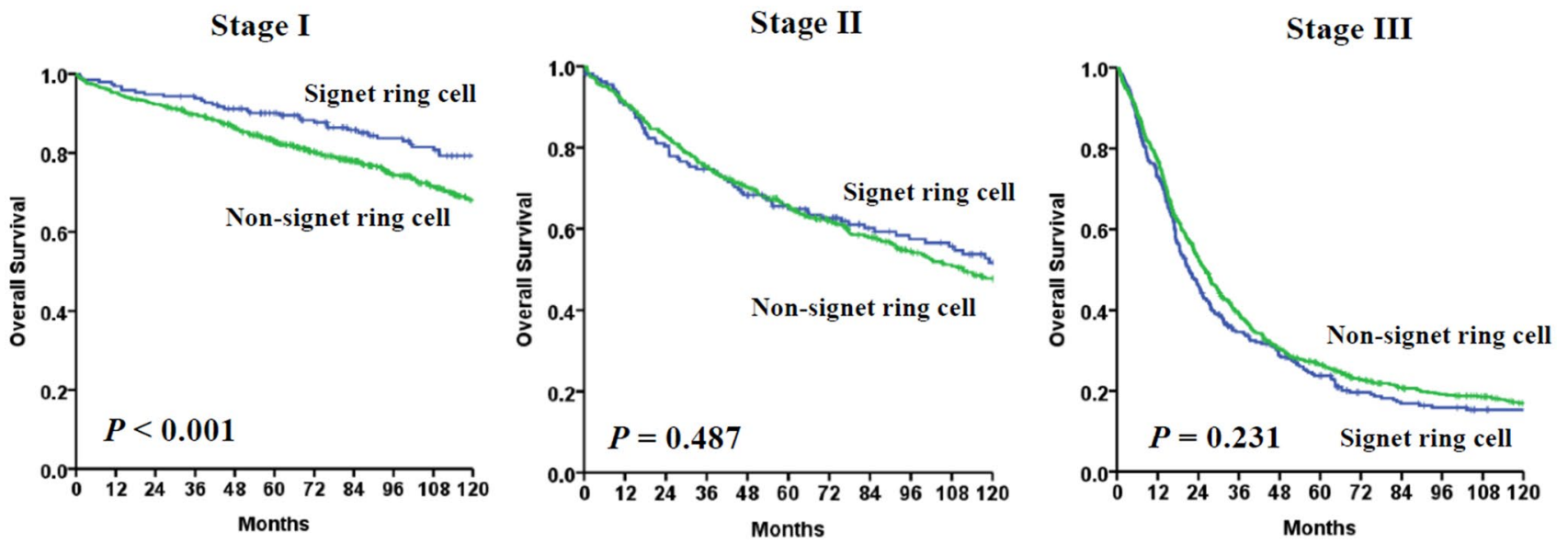

Fig. 3 Survival differences in stage I to stage III gastric cancer patients with or without signet ring cell adenocarcinoma

Table 4 Recurrent rate in gastric cancer with signet ring cell adenocarcinoma

\begin{tabular}{lllrl}
\hline Variables & \multicolumn{2}{l}{ Recurrence } & $P$ value \\
\cline { 2 - 3 } & Positive & Negative & Total & \\
\hline Early gastric cancer & & & & \\
$\quad$ Signet ring cell & 11 & 174 & 185 & \\
$\quad$ Non-signet ring cell & 33 & 601 & 634 & 0.694 \\
Advanced gastric cancer & & & & \\
$\quad$ Signet ring cell & 188 & 382 & 570 & \\
$\quad$ Non-signet ring cell & 379 & 1203 & 1582 & $<0.001^{*}$ \\
Total & 611 & 2360 & 2971 & \\
\hline
\end{tabular}

$* P<0.05$, statistical significance

cell gastric cancer. Previous studies have conflicting results of the prognosis in early and advanced signet ring cell gastric cancer.
Comparing to previous studies, their reports adopted the definition of WHO classification with the definition of more than 50\% signet ring cell in the gastric tumor. However, these studies did not clearly define the percentage of the mucosa part or deep invasive part of signet ring cell. Therefore, the incidence of signet ring cell adenocarcinoma varies from 3.4 to $50 \%$ [22]. Some advanced gastric cancer without the presence of mucosal signet ring cell may be classified into signet ring cell carcinoma. Therefore, these studies may be dubious for the results without clear definition. Based on the definition, our study is a large-scale study that investigated both early and advanced signet ring cell gastric adenocarcinoma which obtained opposite survival results in these two groups.

In terms of prognostic factors, Chon et al. [23] and Jiang et al. [16] reported that signet ring cell adenocarcinoma was an independent prognostic factor for early gastric cancer but not for advanced gastric cancer. Conflicting results on the 
prognostic value of signet ring cell carcinoma have also been reported from several studies [22]. In contrast to their studies, even though the survival of early gastric cancer patients with signet ring cell adenocarcinoma was superior to those with non-signet ring adenocarcinoma, we found that the presence of signet ring cell adenocarcinoma is not a significant prognostic factor for early gastric cancer. Except for the lymph node metastasis status, there seem to be few prognostic factors for early gastric cancer in our series. However, there are many prognostic factors in advanced gastric cancer, including tumor size, Borrmann classification, tumor invasion depth (TNM T category), lymph node metastasis (TNM N category) and the presence of signet ring cell.

Interestingly, we found that patients with signet ring cell gastric cancer had better survival than those with non-signet ring cell gastric cancer in stage I gastric cancer, but not in stage II or stage III gastric cancer, based on the AJCC staging systems (8th edition). Staging variation in signet ring cell gastric cancer was also reported by Bamboat et al. [12]. Though there was no significant difference in stage II and stage III gastric cancer in our series, we found that the survival curves of signet ring cell adenocarcinoma shifted to below those of non-signet ring cell adenocarcinoma. Perhaps, the prognosis of stage II and stage III signet ring cell gastric cancer is not just influenced by the histological pattern. Since AJCC TNM staging is multifactorial, and includes variable $\mathrm{T}$ and $\mathrm{N}$ factors, the tumor invasion depth or lymph node metastases status may play a more important role in stage II and stage III signet ring cell gastric cancer.

The indication for endoscopic submucosal resection for early gastric cancer with signet ring cell adenocarcinoma is currently a critical issue for gastric cancer treatment [17, 24]. The general concept of the indications for treatment of early gastric cancer with signet ring cell adenocarcinoma is a gastric tumor that is limited to mucosal layer and without lymph node metastasis. Lee et al. [25] recommended that endoscopic submucosal resection should be less than $1 \mathrm{~cm}$ for signet ring cell adenocarcinoma and less than $2 \mathrm{~cm}$ for undifferentiated gastric adenocarcinoma. Hyung et al. [18] reported that early gastric cancer had a lower rate of lymph node metastasis. Therefore, less invasive surgery was recommended for early gastric cancer with signet ring cell adenocarcinoma. However, our report showed that the incidence of lymph nodes metastasis is $15.7 \%$ in early gastric cancer with signet ring cell adenocarcinoma, while it was $13.4 \%$ in early gastric cancer with non-signet ring cell adenocarcinoma. Though there were no significant differences between these two groups, we recommend that endoscopic submucosal resection for signet ring cell adenocarcinoma should be limited to selected cases because of the relatively higher of lymph node metastasis in early gastric cancer. In advanced gastric cancer, lymph node metastasis was found in $80.5 \%$ of cases of signet ring cell adenocarcinoma, while $74.1 \%$ of cases of non-signet ring cell adenocarcinoma $(P<0.001)$. Curative gastrectomy with radical lymph node dissection in both signet and non-signet ring cell adenocarcinoma was recommended as therapeutic strategies with curative intent.

There are few literature reports of the recurrence status in signet ring cell gastric cancer. Our series showed that the overall recurrence rates of gastric cancer patients with and without signet ring cell were 26.3 and $18.5 \%$, respectively, $P<0.001$. Patients with signet ring cell adenocarcinoma still had higher recurrent rates. Compared to Chon et al. [23], signet ring cell carcinoma is a borderline predictor of recurrence (DFS: $P=0.066$, hazard ratio: $1.15,95 \%$ confidence interval: 0.99-1.34) for advanced gastric cancer; our study found a significant predictor of recurrence in signet ring cell adenocarcinoma for advanced gastric cancer. In addition, the rates of peritoneal dissemination and distant lymph node metastasis in patients with signet ring adenocarcinoma were significantly higher than in those with non-signet ring cell adenocarcinoma. Based on these results, carefully follow-up examinations and more aggressive treatment may be necessary for signet ring cell adenocarcinoma after surgery. Since 2008, adjuvant oral fluoropyrimidine drug S-1 has been prescribed as a treatment, if it is financially affordable for the patient, for stage II or stage III gastric cancer patients in our hospital after curative resection. Signet ring cell adenocarcinoma used to be associated with less sensitivity to chemotherapy than non-signet ring cell adenocarcinoma [10]. However, the response for chemotherapy in signet ring cell adenocarcinoma is still under debate [26, 27].

This study was a 25 -year experience in a single medical center that was based on retrospective investigations. We enrolled large numbers of gastric cancer patients who underwent curative resection and had complete pathological examinations. However, selection bias might have existed in this retrospective study. In addition, our study observed the clinical feature and patient prognosis in gastric cancer. Investigations of genetic alterations and tumor cell behavior in signet ring cell adenocarcinoma should be designed in the future.

\section{Conclusion}

Early gastric cancer patients with signet ring cell adenocarcinoma had better survival than those with non-signet ring cell adenocarcinoma. Signet ring cell adenocarcinoma was an independent predictor of poor prognosis in advanced gastric cancer after curative resection.

Acknowledgements We thank Miss L-J Dai (Department of Surgery, Taipei Veterans General Hospital) for her assistance with statistics. This research was supported by a grant from Taipei Veterans General Hospital (V107C-043) and grants from the Ministry of Science and Technology (MOST 105-2314-B-075-005-MY2, 107-2314-B-075-005-MY2). 
All sources of funding had no roles in the study design, data collection, analysis and interpretation of data, writing of the manuscript, or in the decision to submit the manuscript for publication.

\section{Compliance with ethical standards}

Ethical standards This study was approved by the Institutional Review Board (IRB-TPEVGH No. 2017-05-004BC) from Taipei Veterans General Hospital.

Conflict of interest All authors disclose no financial and personal relationships with other people or organizations that could inappropriately influence their work.

\section{References}

1. Ferlay J, Soerjomataram I, Dikshit R, Eser S, Mathers C, Rebelo $\mathrm{M}$, et al. Cancer incidence and mortality worldwide: sources, methods and major patterns in GLOBOCAN 2012. Int J Cancer. 2015;1(5):E359-86. 136).

2. Japanese Gastric Cancer Association. Japanese classification of gastric carcinoma, 2nd English ed. Gastric Cancer 1998;1:10-24.

3. Lauren P. The two histologic main types of gastric carcinoma: diffuse and so-called intestinal type carcinoma. An attempt at a histoclinicalclassification. Acta Parhol Microbid Scan. 1965;64:31-49.

4. Typing of Oesophageal and Gastric Tumours. WHO international histological classification of tumours no. 18 (ed 2). Berlin: Springer; 1990

5. Piessen G, Messager M, Leteurtre E, Jean-Pierre T, Mariette C. Signet ring cell histology is an independent predictor of poor prognosis in gastric adenocarcinoma regardless of tumoral clinical presentation. Ann Surg. 2009;250(6):878-87.

6. Li C, Kim S, Lai JF, Hyung WJ, Choi WH, Choi SH, et al. Advanced gastric carcinoma with signet ring cell histology. Oncology. 2007;72(1-2):64-8.

7. Wu CW, Hsiung CA, Lo SS, Hsieh MC, Chen JH, Li AF, et al. Nodal dissection for patients with gastric cancer: a randomized controlled trial. Lancet Oncol. 2006;7:309-15.

8. Ming SC. Gastric carcinoma: a pathological classification. Cancer. 1977;39(6):2475-85.

9. American Joint Committee on Cancer. AJCC cancer staging manual. 8th ed. New York: Springer; 2017.

10. Pernot S, Voron T, Perkins G, Lagorce-Pages C, Berger A, Taieb J. Signet-ring cell carcinoma of the stomach: impact on prognosis and specific therapeutic challenge. World J Gastroenterol. 2015;21(40):11428-38.

11. Kunisaki C, Shimada H, Nomura M, Matsuda G, Otsuka Y, Akiyama $\mathrm{H}$. Therapeutic strategy for signet ring cell carcinoma of the stomach. Br J Surg. 2004;91(10):1319-24.

12. Bamboat ZM, Tang LH, Vinuela E, Kuk D, Gonen M, Shah MA, et al. Stage-stratified prognosis of signet ring cell histology in patients undergoing curative resection for gastric adenocarcinoma. Ann Surg Oncol. 2014;21:1678-85.
13. Kwon KJ, Shim KN, Song EM, Choi JY, Kim SE, Jung HK, et al. Clinicopathological characteristics and prognosis of signet ring cell carcinoma of the stomach. Gastric Cancer. 2014;17:43-53.

14. Maehara Y, Sakaguchi Y, Moriguchi S, Orita H, Korenaga D, Kohnoe S, Sugimachi K. Signet ring cell carcinoma of the stomach. Cancer. 1992;69:1645-50.

15. Kim DY, Park YK, Joo JK, Ryu SY, Kim YJ, Kim SK. el al. Clinicopathological characteristics of signet ring cell carcinoma of the stomach. ANZ J Surg. 2004;74:1060-4.

16. Jiang CG, Wang ZN, Sun Z, Liu FN, Yu M, Xu HM. Clinicopathologic characteristics and prognosis of signet ring cell carcinoma of the stomach: results from a Chinese mono-institutional study. J Surg Oncol. 2011;103(7):700-3.

17. Ha TK, An JY, Youn HK, Noh JH, Sohn TS, Kim S. Indication for endoscopic mucosal resection in early signet ring cell gastric cancer. Ann Surg Oncol. 2008;15(2):508-13.

18. Hyung WJ, Noh SH, Lee JH, Huh JJ, Lah KH, Choi SH, et al. Early gastric carcinoma with signet ring cell histology. Cancer. 2002;94(1):78-83.

19. Gronnier C, Messager M, Robb WB, Thiebot T, Louis D, Luc $\mathrm{G}$, et al. Is the negative prognostic impact of signet ring cell histology maintained in early gastric adenocarcinoma? Surgery. 2013;154:1093-9.

20. Taghavi S, Jayarajan SN, Davey A, Willis AI. Prognostic significance of signet ring gastric cancer. J Clin Oncol. 2012;30:3493-8.

21. Li C, Kim S, Lai JF, Hyung WJ, Choi WH, Choi SH, et al. Advanced gastric carcinoma with signet ring cell histology. Oncology. 2007;72:64-8.

22. Nie RC, Yuan SQ, Li YF, Chen YM, Chen XJ, Zhu BY, et al. Clinicopathological characteristics and prognostic value of signet ring cells in gastric carcinoma: a meta-analysis. J Cancer. 2017;208(17):3396-404.

23. Chon HJ, Hyung WJ, Kim C, Park S, Kim JH, Park CH, et al. Differential prognostic implications of gastric signet ring cell carcinoma: stage adjusted analysis from a single high-volume center in Asia. Ann Surg. 2017;265:946-53.

24. Kim HM, Pak KH, Chung MJ, Cho JH, Hyung WJ, Noh SH, et al. Early gastric cancer of signet ring cell carcinoma is more amenable to endoscopic treatment than is early gastric cancer of poorly differentiated tubular adenocarcinoma in select tumor conditions. Surg Endosc. 2011;25(9):3087-93.

25. Lee IS, Lee S, Park YS, Gong CS, Yook JH, Kim BSl. Applicability of endoscopic submucosal dissection for undifferentiated early gastric cancer: mixed histology of poorly differentiated adenocarcinoma and signet ring cell carcinoma is a worse predictive factor of nodal metastasis. Surg Oncol. 2017 Mar;26(1):8-12.

26. Messager M, Lefevre JH, Pichot-Delahaye V, Souadka A, Piessen G, Mariette C. The impact of perioperative chemotherapy on survival in patients with gastric signet ring cell adenocarcinoma: a multicenter comparative study. Ann Surg. 2011;254:684-93.

27. Heger U, Blank S, Wiecha C, Langer R, Weichert W, Lordick F, et al. Is preoperative chemotherapy followed by surgery the appropriate treatment for signet ring cell containing adenocarcinomas of the esophagogastric junction and stomach? Ann Surg Oncol. 2014;21:1739-48. 